\title{
Unified Error Control Framework with Cross-Layer Interactions for Efficient H.264 Video Transmission over IEEE 802.11e Wireless LAN
}

\author{
Jeong-Yong Choi and Jitae Shin \\ School of Information and Communication Engineering, \\ Sungkyunkwan University, Suwon, 440-746, Korea \\ \{eldragon, jtshin\}@ece.skku.ac.kr
}

\begin{abstract}
Efficient H.264 video transmission, as a dominant video coding standard used in Digital Multimedia Broadcasting (DMB) and other advanced video conferencing, over wireless/mobile networks becomes dominant. However, wireless video transmission suffers from deficient wireless channel conditions such as high bit error rate, error bursts due to channel fading and bandwidth limitations. In this paper, a novel design of unified error-control with cross-layer interaction over IEEE 802.11e Wireless LAN, in order to improve error protection performance, is proposed. This framework combines cross-layer error protection techniques, i.e., error correction code in the link layer, erasure code in the application layer and automatic repeat retransmission across the link layer and the application layer.
\end{abstract}

\section{Introduction}

In Korea, Satellite Digital Multimedia Broadcasting (S-DMB) and Terrestrial Digital Multimedia Broadcasting (T-DMB) services have been widely standardized on a national basis. S-DMB was launched as a test-service in January and as a main-service in May 2005, respectively, and T-DMB commenced service in December 2005. The Korean DMB system 1 adopts the European Digital Audio Broadcasting (DAB) system known as Eureka-147 [2] as its base, and adds various coding, networking and error correcting tools to process multimedia content. While S-DMB and T-DMB services have a different system, the associated video coding techniques are based on the H.264/Advanced Video Coding (AVC) baseline profile [3]. The H.264/AVC video coding standard provides for coding efficiency and network friendliness, splitting the Video Coding Layer (VCL) and Network Adaptation Layer (NAL).

In the trend of broadcasting communication convergence, multimedia content is supplied via various communication channels. IEEE 802.11-based Wireless LANs (WLANs) can be one a great communication channel, supplying users with portability. IEEE 802.11e [4] has been developed and standardized to support QoS of Audio/Visual (AV) data. In contrast to wired networks, since wireless 
channels suffer from restrictions such as bit-error and bandwidth, video transmission over wireless channels is a challenging task.

There has been considerable research invested, to overcome such inferior channel conditions. Many hybrid ARQ (HARQ) techniques combine various errorcontrol schemes [5] [6. They include error detection code, Forward Error Correction (FEC) and Automatic Repeat reQuest (ARQ). Although aforementioned error-control-related research proposes efficient error protection schemes, there have been few concrete cross-layer frameworks in WLANs.

In this paper, a novel cross-layer error-control framework in IEEE 802.11e wireless LAN is proposed to improve error protection performance. This framework combines cross-layer error protection schemes, i.e., error correction code (Reed-Solomon code) in the link layer, erasure code in the application layer and ARQ across both the link layer and the application layer.

The remainder of this paper is organized as follows. A brief overview of H.264 video transmission in wireless channels and a review of error-control techniques are provided in Section 2 Section 3 describes the proposed unified cross-layer error-control framework. In Section 4, the performance of proposed framework is evaluated. Lastly, conclusions and further works are presented in Section 5 .

\section{Related Works on Key Error-Control Components}

\subsection{Error-Controls for Video Transmission}

FEC and delay-constrained ARQ are the main error-control techniques in video transmission. FECs, which are based both on the error correction code and on the erasure code, realize a proactive error-control mechanism. The sender generates redundant data and transmits these data with the original data. Then, the receiver recovers the erroneous bits or packets with redundant data. In order to prevent confusion, FECs are classified into two categories, i.e., error correction code-based FEC (namely Symbol-level FEC, S-FEC) and erasure code-based FEC (namely Packet-level FEC, P-FEC). While both S-FEC and P-FEC provide proactive solutions, ARQ is driven by reactive feedback information. ARQ spends less overhead in comparison with constantly rate-wasteful FECs, because ARQ is driven only when the received packet is erroneous, ARQ tends to result in greater delay than FECs.

In order to alleviate error resilience, hybrid techniques were attempted, and type-I [7] and type-II HARQ $[8$ ] were introduced. H. Liu et al. proposed a HARQ technique that combines the benefit of type-I and type-II techniques, and showed its efficacy for video transmission over wireless networks [5]. Y. Shan et al. presented a priority-based error protection scheme in the sense of the cross-layer approach [6]. Lastly, P. Ferré et al. attempted to modify the IEEE 802.11 legacy MAC to improve the throughput efficiency [9. The article includes composition of an MAC frame and modification of MAC-level retransmission.

This paper is dominantly motivated by [9, to move the wireless channel to IEEE 802.11e, and makes use of the FEC option and BlockAck mechanism of IEEE 802.11e MAC. 


\subsection{IEEE 802.11e MAC-Level Error Control Techniques}

Symbol-Level Forward Error Correction (S-FEC) [10]. Fig. 11 shows the MAC Protocol Data Unit (MPDU) format defined in the draft specification of IEEE 802.11e. Basically, a $(224,208)$ shortened Reed Solomon (RS) code, defined in GF(256), is used. Since an MAC Service Data Unit (MSDU), from the higher layer, can be much larger than 208 octets, an MSDU may be split into (up to 12) multiple blocks, and then each block is separately encoded by the RS encoder. The final RS block in the frame body can be shorter than 224 octets, using a shortened code. A $(48,32) \mathrm{RS}$ code, which is also a shortened RS code, is used for the MAC header, and CRC-32 is used for the Frame Check Sequence (FCS). It is important to note that any RS block can correct up to 8 byte errors. The outer FCS allows the receiver to skip the RS decoding process if the FCS is correct. The inner FCS (or FEC FCS) allows the receiver to identify a false decoding by the RS decoder.

\begin{tabular}{|c|c|c|c|c|c|c|c|c|c|}
\hline \multicolumn{2}{|c|}{ MAC Header } & \multicolumn{7}{|c|}{$\begin{array}{c}\text { Frame Body } \\
\text { ( } N \text { Blocks) }\end{array}$} & \multirow{2}{*}{\begin{tabular}{|l} 
FCS \\
FCS
\end{tabular}} \\
\hline Header & $\begin{array}{l}\text { Header } \\
\text { FEC }\end{array}$ & $\mathrm{MSDU}_{1}$ & FEC & $\mathrm{MSDU}_{2}$ & FEC & $\ldots$ & $\begin{aligned} & \text { MSDUN }_{N} \\
+ & \text { FEC FCS }\end{aligned}$ & FEC & \\
\hline 32 & 16 & 208 & 16 & 208 & 16 & $\ldots$ & 208 & 16 & 4 \\
\hline
\end{tabular}

Fig. 1. IEEE 802.11e MPDU format with the optional FEC

ARQ [11]. The legacy MAC of IEEE 802.11 is based on the simple Stop-andWait ARQ (SW-ARQ) scheme. This involves a lot of overheads due to the immediate transmissions of acknowledgements (ACKs). In 802.11e, a new SelectiveRepeat ARQ (SR-ARQ) mechanism named block acknowledgement (BlockAck) is introduced. In this mechanism, a group of data frames can be transmitted one by one with SIFS interval between them. Then, a single BlockAck frame is transmitted back to the sender to inform ACKs how many packets have been received correctly. Obviously, this scheme can improve channel efficiency. There are two kinds of BlockAck mechanisms used in 802.11e: immediate and delayed. Details of each modes are presented in [11. In this paper, the delayed BlockAck mechanism will be adopted between two mechanisms, so as to satisfy the requirements of delayed operations across layers.

\section{Unified Cross-Layer Cooperating Error-Control Framework}

In this section, a unified error-control framework with cross-layer interactions is proposed, and the role of each layer and cross-layer cooperation are described. The overall structure of the proposed framework is depicted in Fig. $2^{1}$ The

\footnotetext{
${ }^{1}$ Since, in this paper, the range of the implementation is restricted, the implemented components are marked as shadow in Fig. 3
} 


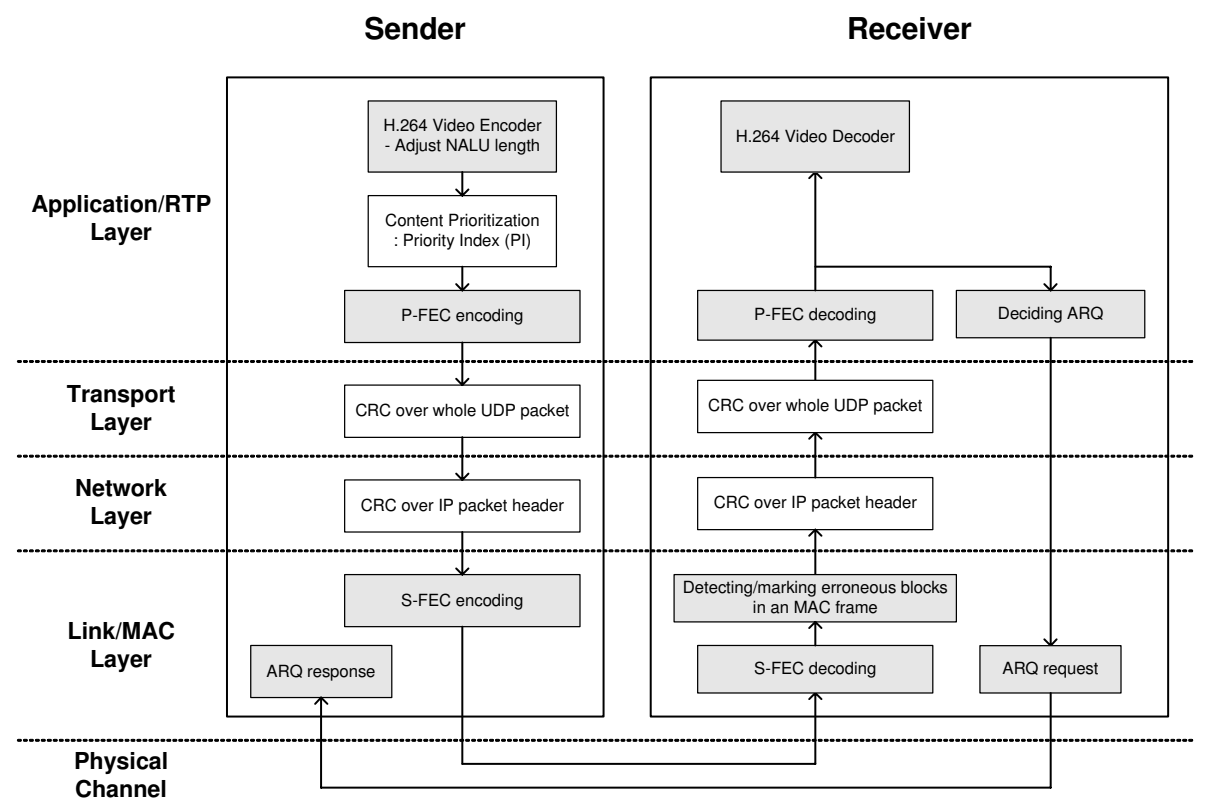

Fig. 2. Overall structure of the unified error-control framework with cross-layer interactions

techniques and schemes previously explained in Section 2 are combined, in order to achieve an efficient framework.

\subsection{Link/MAC Layer}

As explained in 2.2, the link/MAC layer performs S-FEC. On the sender side, the MAC header and all MSDUs are encoded using $(48,32)$ and $(224,208)$ $\mathrm{RS}$ codes, respectively, and then composed into an MAC frame. After S-FEC encoding, the whole MAC frame is calculated and the CRC-32 and FCS field are generated.

On the receiver side, prior to S-FEC decoding, the FCS check is performed. If the FCS is correct, the S-FEC decoding procedure is unnecessary, and is therefore not performed. However, if the FCS is incorrect, all S-FEC blocks are decoded and erroneous blocks are corrected. If MAC header is erroneous and uncorrectable, all of the MSDUs belonging to the MAC header will be dropped. If an FEC block of MSDUs is uncorrectable, the block is marked as erroneous and then passed to a higher layer. The uncorrectable S-FEC block is determined whether to be retransmitted in the application layer.

In addition to S-FEC, the link/MAC layer provides ARQ scheme for the erroneous MSDUs, as explained in 2.2. Since the uncorrectable S-FEC block is passed to higher layers, not immediately requested for retransmission, the delayed BlockAck scheme is used, rather than the immediate BlockAck scheme. 
The sequence numbers of the uncorrectable S-FEC blocks determined to be retransmitted are bitmapped in the BlockAck frame.

\subsection{Application Layer}

In the proposed cross-layer framework, the application layer is responsible for packet-level adaptation and cross-layer interaction. Packet-level adaptation includes H.264 encoding, priority indexing (PI), RTP packetization and P-FEC. In this procedure, the H.264 encoder should consider the maximum length of an MSDU at the link/MAC layer. This implies that if one RTP packet is not fragmented into greater than one MSDU at the link/MAC layer, so as not to deteriorate error resilience, the maximum length of an RTP packet, including 2-byte P-FEC header, should not exceed 180 bytes, as presented in Fig. 3. Sizeadjusted H.264 NAL Units (NALUs) are P-FEC-encoded and packetized into an RTP packet. Priorities of each NALUs are estimated and indexed, while NALUs are simultaneously encoded. The PI method which is used in [12] may be extended for H.264 2 The PI-values can be criteria for UEP strategy.

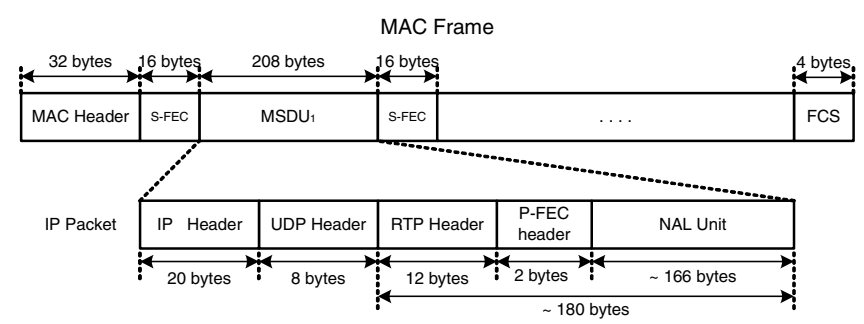

Fig. 3. Structure of an MAC frame

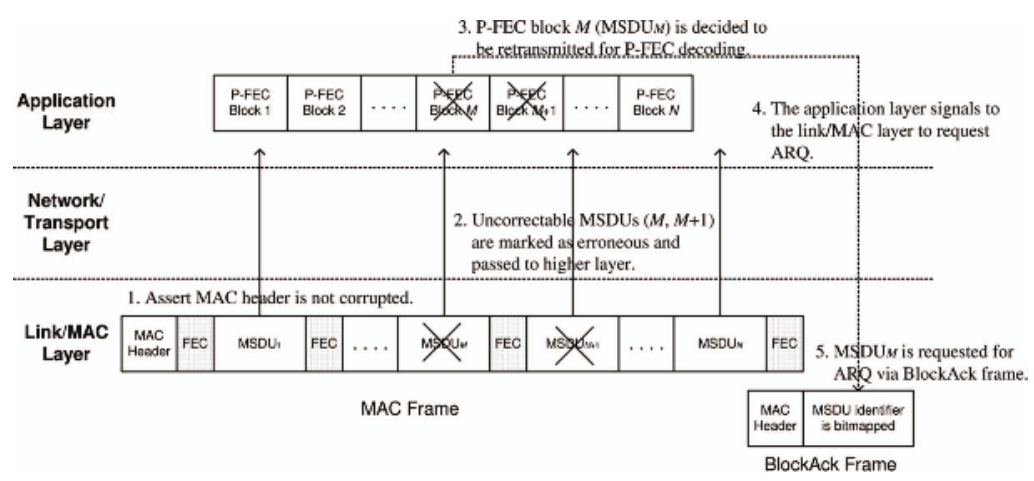

Fig. 4. Cross-layer error-control process at the receiver

${ }^{2}$ Although we include PI in the proposed framework, it is out of scope in this paper because of page limit. 
Next, consider the cross-layer interaction. The proposed framework complements S-FEC with P-FEC and ARQ. First, S-FEC protects all symbols in a packet. Next, if S-FEC proves to be uncorrectable, P-FEC recovers the erroneous data blocks. Lastly, if P-FEC fails, the application layer signals to the link layer to request for retransmission of the necessary data blocks, to ensure that cross-layer cooperation is completed. The detailed error-control process is depicted and explained in Fig. 4. To conclude, the application layer adapts the video content to the underlying structure of the link/MAC layer, and decides the error-control strategy, whereas the link/MAC layer provides the application layer with the transport information of the packets and a means for retransmission.

\section{Simulation}

In this section, the performance of the proposed cross-layer error-control framework is demonstrated over IEEE 802.11e WLAN, by performing simulations under several BER conditions. The "Foreman" sequence (CIF, 352x288) was encoded using H.264 with encoding parameters, as presented in Table 11 and then transmitted through the Gilbert-Elliott channel model. The performance of the generic error-control scheme and the proposed error-control scheme are compared. Both schemes consist of $(24,20)$ P-FEC at the application layer, (224, 208) S-FEC at the link/MAC layer and ARQ with maximum ARQ count of 2.

Table 1. Video encoder parameters and channel settings used in the simulation

\begin{tabular}{l|l}
\hline \hline Parameter settings \\
\hline \hline Video encoder & H.264 (JM 10.1) \\
Sequence name & Foreman \\
Image format & CIF $(352 \times 288)$ \\
Number of encoded frames & 1200 \\
Encoding method & 1 IDR-frame followed by 1199 P-frames with CBR \\
& (384kbps) 20 random intra MB update every P-frame, \\
& fixed packet size shorter than 180 bytes. \\
\hline \hline Channel settings & Gilbert-Elliott channel model \\
\hline Channel model & $5 \times 10^{-3}$ \\
Bit error probability &
\end{tabular}

Table. 2] and Fig. 5]present the simulation results for the generic isolated-layer scheme and the proposed cross-layer scheme. Table. 2 presents the MSDU overhead rate needed for ARQ and the NALU error rate. From numerical results, it is demonstrated that the proposed cross-layer scheme results in less NALU error rate with dramatically less overhead. The simulation result, presented in Fig. 5], shows that the proposed cross-layer error-control scheme outperforms the isolated-layer error-control scheme, with regard to end-quality. In the isolatedlayer scheme, since even an uncorrectable MSDU in the MAC frame results in 
Table 2. MSDU overhead rate for ARQ and NALU error rate

\begin{tabular}{l|c|c}
\hline & Isolated-layer scheme & Proposed cross-layer scheme \\
\hline MSDU overhead rate for ARQ (\%) & 32.84 & 0.29 \\
\hline NALU error rate (\%) & 0.40 & 0.33 \\
\hline
\end{tabular}

retransmission of the whole MAC frame, the isolated-layer scheme experiences higher bit error probability than the cross-layer scheme. Thus, greater retransmission overhead is required, and high MAC frame error probability deteriorates throughput, eventually, producing degradation of objective video quality.

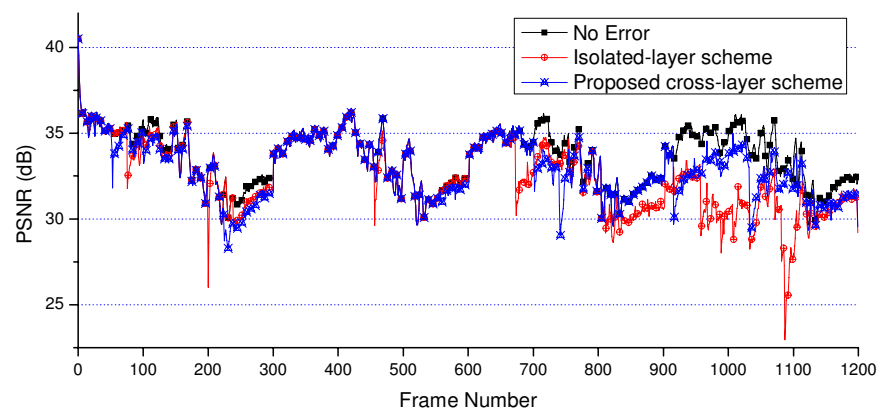

Fig. 5. PSNR distribution of Y-component of transmitted sequences: (a) No error, (b) Isolated-layer error-control scheme, and (c) Proposed cross-layer error-control scheme

From simulation results, it can be inferred that the proposed cross-layer errorcontrol scheme presents excellent performances from the viewpoint of both endquality and transmission efficiency.

\section{Conclusions and Future Works}

In this paper, a unified cross-layer error-control framework was proposed, and the performance of the framework demonstrated. In the proposed error-control framework, each layer does not perform its dedicated role but also considers overall layers, thus unnecessary operations can be omitted. From simulation results, it can be inferred that the proposed cross-layer error-control scheme demonstrates excellent performance from the viewpoint of both end-quality and transmission efficiency.

FEC and ARQ have been the most powerful error-control tools in data communication. In order to maximize the performance of FEC and ARQ, combined with packet-level interleaving and symbol-level interleaving can be considered. In current work, we cannot present the mathematical analysis of the proposed scheme, because of page limit. Mathematical analysis under certain parameters of Gilbert-Elliott channel helps to estimate the simulation result. 


\section{Acknowledgements}

This research was supported by the Ministry of Information and Communication (MIC), Korea, under the Information Technology Research Center (ITRC) support program supervised by the Institute of Information Technology Assessment (IITA) (IITA-2005-(C1090-0502-0027)).

\section{References}

1. "Digital Multimedia Broadcasting," Telecommunications Technology Association, 2003SG05.02-046, 2003.

2. "Radio Broadcasting System: Digital Audio Broadcasting (DAB) to mobile, portable and fixed receivers," ETSI EN 300401 v1.3.3, May, 2001.

3. Draft ITU-T Recommendation and Final Draft International Standard of Joint Video Specification (ITU-T Rec. H.264 - ISO/IEC 14496-10 AVC), Document: JVT-G050r1, May. 2003.

4. IEEE 802.11e/D3.0, "Draft Supplement to Part 11: Wireless Medium Access Control (MAC) and Physical Layer (PHY) specifications: Medium Access Control (MAC) Enhancements for Quality of Service (QoS)," May, 2002.

5. H. Liu and E. Zarki, "Performance of H.263 Video Transmission over Wireless Channels Using Hybrid ARQ," IEEE Journal on Selected Areas in Communications, Vol. 15, No. 9, Dec. 1997.

6. Y. Shan and A. Zakhor, "Cross Layer Techniques for Adaptive Video Streaming over Wireless Networks," International Conference on Multimedia and Expo, Lausanne, Switzerland, Aug. 2002, pp. 277-280.

7. H. Deng and M. Lin, "A Type I Hybrid ARQ System with Adaptive Code Rates," IEEE Trans. Communications, vol. 46, pp. 733-737, Feb. 1995.

8. S. Lin and D. Costello, "Error Control Coding: Fundamentals and Applications," Englewood Cliffs, NJ: Prentice-Hall, 1983.

9. P. Ferré, A. Doufexi, A. Nix, D. Bull, and J. Chung-How, "Packetisation Strategies for Enhanced Video Transmission over Wireless LANs," Packet Video Workshop (PV2004), Irvine, CA, USA, 1314 Dec. 2004.

10. S. Choi, "IEEE 802.11e MAC-Level FEC Performance Evaluation and Enhancement," IEEE Global Telecommunications Conference, 2002.

11. Q. Ni, L. Romdhani and T. Turletti, "A Survey of QoS Enhancements for IEEE 802.11 Wireless LAN," RFC 3550, IETF, Jul. 2003.

12. J. Shin, J. G. Kim, J. W. Kim and C.-C.J. Kuo, "Dynamic QoS mapping control for streaming video in relative service differentiation networks," European Transactions on Telecommunications, Vol. 12, No. 3, May-June 2001, pp. 217-230. 